\title{
Spectrum Sharing in Cognitive Radio with Quantized Channel Information
}

\author{
YuanYuan He, Subhrakanti Dey and Vasanthan Raghavan \\ Department of Electrical and Electronic Engineering, The University of Melbourne \\ \{yyhe, s.dey\}@ee.unimelb.edu.au, vasanthan_raghavan@ieee.org
}

\begin{abstract}
In this paper, we consider a wideband spectrum sharing system where a secondary user can share orthogonal frequency bands with a number of primary users, each licensed to use an individual band. We address the problem of ergodic capacity maximization of the secondary user subject to an average sum (across the bands) transmit power constraint on the secondary user and individual average interference constraints on the primary users. After studying optimal power allocation with full channel state information (CSI) at the secondary transmitter, we focus on designing optimal power allocation strategies for the quantized CSI case. It is assumed that a band manager has the full CSI of all secondary-to-secondary and secondary-to-primary channels, and designs (ofine) an optimal power codebook based on statistical information of the channels and feeds back the index of the codebook to the secondary transmitter for every channel realization. A modified generalized Lloyd type algorithm (GLA) is designed for an optimal power codebook. With the narrowband case (one secondary and one primary user) in mind, various properties of the quantized powers are derived. It is observed that only 2-3 bits of feedback with this codebook is sufficient to provide secondary capacities close to the full CSI case.
\end{abstract}

\section{INTRODUCTION}

Radio spectrum is a limited and precious resource that is traditionally licensed to users by governments in a rigid manner. In order to avoid interference, the licensed owner has an exclusive right to access the allocated frequency band [1]. Consequently, as the number of wireless communication systems and services grow, the availability of vacant spectrum becomes severely scarce. However, some recent measurements reveal that many portions of the spectrum are mostly under-utilized or even unoccupied. This has led to the idea of cognitive radio (CR) technology [2], which holds tremendous promise to dramatically improve the spectral efficiency. The key idea behind CR is that an secondary user (SU) is allowed to communicate over the frequency band originally licensed to a primary user (PU), as long as the transmission of SU does't generate unfavorable impact on the operation of PU.

Basically, there are three categories of CR network paradigms: interweave, overlay, and underlay [3]. In underlay systems, the SU can transmit even when the PU is present, but the transmitted power of SU should be controlled properly so as to ensure that the resulting interference does not degrade the received signal quality of PU to an undesirable level [6] by imposing the interference temperature [1] constraint at PU (average or peak interference power (AIP/PIP) constraint). This type of CR is also known as 'spectrum sharing' [1] model. In this paper, we will focus on this underlay (spectrum sharing) model.

The behavior of capacities of different AWGN channels under received-power constraints (AIP) at the PU receiver (PU-RX) is studied in [7]. Lack of fading results in the almost similar capacity performance with transmit or received power constraints. The ergodic capacity of narrowband spectrum sharing model with one SU and one or more PU under either AIP or PIP constraint at PU-RX under various fading distributions is studied in [1], illustrating that with fading, spectrum access opportunity for the SU significantly increases compared to the AWGN case. In [9], optimum power allocation for three different capacity notions under both AIP and PIP constraints is studied. The optimal power transmission strategies for maximizing ergodic capacity and outage capacity under various combinations of secondary transmit power constraints and interference constraints is designed in [6].

However, most of the above results assume to have knowledge of full channel state information (CSI) including the SUTX to PU-RX channels, which is hard to realize in practice. Recently, the effect of imperfect channel estimation in the secondary to primary channels has been investigated in [10] by considering the channel estimate as a noisy version of the true CSI, and [16] proposed a practical design paradigm for cognitive beamforming based on finite-rate cooperative feedback from the PU-RX to the SU-TX. In this paper, we consider a wideband spectrum sharing system where one SU shares $M$ different frequency bands with $M$ primary users (PUs), each using a separate band. Under the general framework of limited feedback, we address the problem of ergodic capacity maximization of SU subject to an average sum (across the bands) transmit power constraint on the SU and individual average interference constraints on the PUs.

After presenting a systematic algorithm for optimal power allocation with full CSI at the secondary transmitter, we turn to the main focus of this paper - designing optimal power allocation using quantized channel information. It is assumed that a band manager or CR service provider has full CSI including all secondary-to-secondary and secondaryto-primary channels. It designs an optimal power codebook based on the statistical information (channel distributions) of the channels and feeds back the index of the codebook to the secondary transmitter for every channel realization. A generalized Lloyd type algorithm (GLA) is designed for the optimal power codebook, which is globally convergent and empirically consistent. Focusing on the narrowband case (one secondary and one primary user), various properties of the quantized powers are derived. Numerical studies illustrate that only 2-3 bits of feedback provide secondary capacities very close to capacity based on full CSI.

\section{System Model And PRoblem Formulation}

We consider a wideband spectrum sharing scenario with one SU and Multiple PUs, where a SU is allowed to use $M$ orthogonal frequency bands $\left(\operatorname{Band}_{1}\right.$ to $\left.\operatorname{Band}_{M}\right)$ that are individually licensed to $P U_{1}, \ldots, P U_{M}$, respectively. Regardless of the ON/OFF status of $P U_{i}$, SU uses the $i$-th channel as long as the impact of the secondary transmission does not substantially degrade the received signal quality of $P U_{i}$. Let $g_{0}^{i} \in \mathbb{R}_{+}$and $g_{1}^{i} \in \mathbb{R}_{+}$denote the instantaneous channel power gains of the link between the SU-TX and the receiver of $P U_{i}$ and $i$-th channel between the SU-TX and 
SU-RX, respectively. These channels are assumed to be block fading channels with probability density functions $f_{0}\left(g_{0}^{i}\right)$ and $f_{1}\left(g_{1}^{i}\right)$. For analytical simplicity, the interference from $P U_{i^{-}}$ TX to SU-RX is neglected (as in [1], [6], etc). All $g_{0}^{i}$ and $g_{1}^{i}$ $(i=1, \ldots, M)$ are mutually independent and are assumed to have unit mean. Independent, standard white Gaussian noise is added at each receiver. When $M=1$, this system becomes a typical narrowband spectrum sharing model studied in [1], [5], [6].

Given a channel realization $\mathbf{g}_{0} \triangleq\left\{g_{0}^{1}, \ldots, g_{0}^{M}\right\}$ and $\mathbf{g}_{1} \triangleq$ $\left\{g_{1}^{1}, \ldots, g_{1}^{M}\right\}$, we assume that CSI in the form of $\eta\left(\mathbf{g}_{0}, \mathbf{g}_{1}\right)$ is available at the SU-TX. The power allocated at the SUTX on the $M$ parallel SU links is represented by the vector $\mathbf{p}\left(\eta\left(\mathbf{g}_{0}, \mathbf{g}_{1}\right)\right)=\left\{p_{1}\left(\eta\left(\mathbf{g}_{0}, \mathbf{g}_{1}\right)\right), \ldots, p_{M}\left(\eta\left(\mathbf{g}_{0}, \mathbf{g}_{1}\right)\right)\right\}$. Thus, the ergodic capacity ${ }^{1}$ of the $\mathrm{SU}$ is given as

$$
C=\frac{1}{M} \sum_{i=1}^{M} E\left[\log \left(1+g_{1}^{i} p_{i}\left(\eta\left(\mathbf{g}_{0}, \mathbf{g}_{1}\right)\right)\right)\right]
$$

A common way to protect PU's received signal quality is by imposing either an average or a peak interference power (AIP/PIP) constraint at PU-RX [1], [5], [6], although other forms of PU quality of service constraint such as PU's capacity loss and PU's outage probability [17] can also be used. It was shown in [5] that an AIP constraint is more favorable than a peak constraint especially in the context of transmission over fading channels, since the AIP constraint is more flexible and can achieve larger SU capacity results with less PU capacity loss than those achieved by PIP.

Motivated by this observation, we consider the following optimal power allocation scheme that maximizes the ergodic capacity of SU in a wideband spectrum sharing scenario, under an AIP constraint at each $P U_{i}$-RX and an average sum transmit power constraint (ATP) for the SU, given by,

$$
\begin{gathered}
\underset{p_{i}\left(\eta\left(\mathbf{g}_{0}, \mathbf{g}_{1}\right)\right) \geq 0, \forall i}{\max } \quad \frac{1}{M} \sum_{i=1}^{M} E\left[\log \left(1+g_{1}^{i} p_{i}\left(\eta\left(\mathbf{g}_{0}, \mathbf{g}_{1}\right)\right)\right)\right] \\
\text { s.t. } \quad E\left[g_{0}^{i} p_{i}\left(\eta\left(\mathbf{g}_{0}, \mathbf{g}_{1}\right)\right)\right] \leq Q_{a v, i}, \quad \forall i \\
\frac{1}{M} \sum_{i=1}^{M} E\left[p_{i}\left(\eta\left(\mathbf{g}_{0}, \mathbf{g}_{1}\right)\right)\right] \leq P_{a v} .
\end{gathered}
$$

In Sec. III, we present the optimal power allocation results assuming that full channel state information (CSI) is available at the SU-TX (i.e, $\eta\left(\mathbf{g}_{0}, \mathbf{g}_{1}\right)=\left(\mathbf{g}_{0}, \mathbf{g}_{1}\right)$ ), followed by the case of quantized channel information in Sec. IV, where $\eta\left(\mathbf{g}_{0}, \mathbf{g}_{1}\right)$ represents a deterministic index mapping scheme, such that $\eta\left(\mathbf{g}_{0}, \mathbf{g}_{1}\right)=j, j \in\{1,2, \ldots, L\}, L=2^{B}$ when the instantaneous channel gains $\left(\mathbf{g}_{0}, \mathbf{g}_{1}\right)$ belong to a carefully constructed partition $\mathcal{R}_{j}$ of the channel space $\mathbb{R}_{+}^{M} \times \mathbb{R}_{+}^{M}$. All proofs are relegated to [8] due to paucity of space.

\section{Optimal Power Allocation with Perfect CSI}

In this section, we assume that SU-TX has perfect knowledge of $\mathbf{g}_{0}$ and $\mathbf{g}_{1}$ (full CSI at the transmitter). That is, $\eta\left(\mathbf{g}_{0}, \mathbf{g}_{1}\right)=\left(\mathbf{g}_{0}, \mathbf{g}_{1}\right)$. It is easy to verify that the problem given in (2) is a convex optimization problem. By applying the necessary and sufficient Karush-Kuhn-Tucker (KKT)

\footnotetext{
${ }^{1}$ All capacity expressions are in nats/channel use in this work.
}

conditions for optimality, the optimal power allocation can be easily shown to be

$$
p_{i}^{*}\left(\mathbf{g}_{0}, \mathbf{g}_{1}\right)=\left(\frac{1}{\lambda^{f}+\mu_{i}^{f} g_{0}^{i}}-\frac{1}{g_{1}^{i}}\right)^{+}
$$

where $\lambda^{f}$ and $\mu_{i}^{f}$ are the nonnegative Lagrange multipliers associated with the ATP constraint and the AIP constraint of $P U_{i}$ respectively, and $(x)^{+}=\max (x, 0)$. This solution is clearly a minor extension of the narrowband result in [6]. However, in the wideband case $(M>1)$, it should be noted that determining the optimal power allocation scheme involves obtaining the optimal values of the $(M+1)$ Lagrange multipliers. Since all the constraints in Problem (2) may not hold with equality simultaneously, it is difficult to determine $\lambda^{f}$ and $\left\{\mu_{i}^{f}\right\}$. For example, the Lagrange multipliers can be obtained by the ellipsoid method [15], but this procedure is time consuming. Thus we present a complete solution to Problem (2) in the following theorem.

Theorem 1: With $\eta\left(\mathbf{g}_{0}, \mathbf{g}_{1}\right)=\left(\mathbf{g}_{0}, \mathbf{g}_{1}\right)$ at the SU-TX, the optimal power allocation for Problem (2), $p_{i}^{*}\left(\mathbf{g}_{0}, \mathbf{g}_{1}\right)$, is given by

$$
\begin{aligned}
& \left(\left(\frac{1}{\mu_{i}^{f} g_{0}^{i}}-\frac{1}{g_{1}^{i}}\right)^{+} \Longleftrightarrow P_{a v} \geq \frac{1}{M} \sum_{i=1}^{M} E\left[\left(\frac{1}{\mu_{i} g_{0}^{i}}-\frac{1}{g_{1}^{i}}\right)^{+}\right]\right. \\
& \text {Otherwise } \\
& \begin{cases}\left(\frac{1}{\lambda^{f}}-\frac{1}{g_{1}^{i}}\right)^{+} & \Longleftrightarrow E\left[\left(\frac{1}{\lambda^{f}}-\frac{1}{g_{1}^{i}}\right)^{+}\right] \leq Q_{a v, i} \\
\left(\frac{1}{\lambda^{f}+\mu_{i}^{f} g_{0}^{i}}-\frac{1}{g_{1}^{i}}\right)^{+} & \Longleftrightarrow E\left[\left(\frac{1}{\lambda^{f}}-\frac{1}{g_{1}^{i}}\right)^{+}\right]>Q_{a v, i}\end{cases}
\end{aligned}
$$

For a proof of this result, and associated special cases and numerical studies, see [8]. Appealing to the convexity of Problem (2), we can show that one of the cases must hold, and the corresponding power allocation scheme must be the global optimal solution for the original Problem (2). An algorithm can then be easily designed to obtain $p_{i}^{*}\left(\mathbf{g}_{0}, \mathbf{g}_{1}\right)$, and the associated non-zero Lagrange multipliers can be obtained by solving the KKT optimality conditions numerically (e.g., via a bisection search).

\section{Power Control With QuAntized CSI}

The assumption of full CSI at the SU-TX (especially that of $\mathbf{g}_{0}$ ) is unrealistic in practical systems. In this section, we are therefore interested in designing power allocation schemes based on quantized $\left(\mathbf{g}_{0}, \mathbf{g}_{1}\right)$ information acquired via a no-delay and error-free feedback link with limited rate. We assume that there is a CR service provider or a band manager [4] that can obtain perfect information on $\mathbf{g}_{1}$ from SU$\mathrm{RX}$ or SU base stations and perfect information on $\mathbf{g}_{0}$ from PU base stations, presumably over a wired link, and then forward some appropriately quantized CSI to SU-TX (and SU-RX for decoding purposes) through the feedback link. More specifically, given $B$ bits of feedback, a power codebook $\mathcal{P}=\left\{\mathbf{P}_{1}, \ldots, \mathbf{P}_{L}\right\}$ (where $\mathbf{P}_{j}=\left\{p_{1 j}, \ldots, p_{M j}\right\}, \quad j=$ $1, \ldots, L)$ of cardinality $L=2^{B}$, is designed offline purely on the basis of the statistics of $\mathbf{g}_{0}, \mathbf{g}_{1}$. This codebook is known a priori by both SU-TX and SU-RX. The vector space of $\left(\mathbf{g}_{0}, \mathbf{g}_{1}\right)$ is thus partitioned into $L$ regions $\mathcal{R}_{1}, \ldots, \mathcal{R}_{L}$ using a quantizer $\mathcal{Q}$ (codebook element $\mathbf{P}_{j}$ represents the power level used in $\mathcal{R}_{j}$ ). The $\mathrm{CR}$ service provider/band manager 
maps the current instantaneous $\left(\mathbf{g}_{0}, \mathbf{g}_{1}\right)$ information into one of $L$ integer indices and sends the corresponding index to the SU-TX via the feedback link (e.g., if the current $\left(\mathbf{g}_{0}, \mathbf{g}_{1}\right)$ falls in $\mathcal{R}_{j}$, then $\eta\left(\mathbf{g}_{0}, \mathbf{g}_{1}\right)=j$ will be conveyed back to SU-TX). The SU-TX will use the associated power codebook element (e.g., if the feedback signal is $j$, then $\mathbf{P}_{j}$ will be used as the transmission power) to adapt its transmission strategy.

Let $\operatorname{Pr}\left(\mathcal{R}_{j}\right)=\operatorname{Pr}\left(\left(\mathbf{g}_{0}, \mathbf{g}_{1}\right) \in \mathcal{R}_{j}\right)$ denote the probability that $\left(\mathbf{g}_{0}, \mathbf{g}_{1}\right)$ falls in the region $\mathcal{R}_{j}$. Let $E\left[\bullet \mid \mathcal{R}_{j}\right]=$ $E\left[\bullet \mid\left(\mathbf{g}_{0}, \mathbf{g}_{1}\right) \in \mathcal{R}_{j}\right]$ denote the corresponding expectation. Then, the secondary ergodic capacity maximization problem (2) with limited feedback can be formulated as

$$
\begin{aligned}
\max _{\mathbf{P}_{j} \geq 0, \forall j} & \sum_{j=1}^{L}\left(\frac{1}{M} \sum_{i=1}^{M} E\left[\log \left(1+g_{1}^{i} p_{i j}\right) \mid \mathcal{R}_{j}\right]\right) \operatorname{Pr}\left(\mathcal{R}_{j}\right) \\
\text { s.t. } & \sum_{j=1}^{L} E\left[g_{0}^{i} p_{i j} \mid \mathcal{R}_{j}\right] \operatorname{Pr}\left(\mathcal{R}_{j}\right) \leq Q_{a v, i} \forall i \\
& \sum_{j=1}^{L}\left(\frac{1}{M} \sum_{i=1}^{M} p_{i j}\right) \operatorname{Pr}\left(\mathcal{R}_{j}\right) \leq P_{a v} .
\end{aligned}
$$

Our objective is thus the joint optimization of the channel partition regions and the power codebook such that the ergodic capacity of SU is maximized under the above average transmit power and average interference constrains.

\section{A. Narrowband spectrum-sharing case}

For ease of exposition, we first look at the relatively simpler case of $M=1$. For simplicity (with some abuse of notation), let $p_{j}, g_{1}, g_{0}, Q_{a v}$ represent $p_{1 j}, g_{1}^{1}, g_{0}^{1}, Q_{a v, 1}$ respectively. Thus Problem (4) with $M=1$ is written as

$$
\begin{aligned}
& \max _{p_{j} \geq 0, \forall j} \sum_{j=1}^{L} E\left[\log \left(1+g_{1} p_{j}\right) \mid \mathcal{R}_{j}\right] \operatorname{Pr}\left(\mathcal{R}_{j}\right) \\
& \text { s.t. } \quad \sum_{j=1}^{L} E\left[g_{0} p_{j} \mid \mathcal{R}_{j}\right] \operatorname{Pr}\left(\mathcal{R}_{j}\right) \leq Q_{a v}, \sum_{j=1}^{L} p_{j} \operatorname{Pr}\left(\mathcal{R}_{j}\right) \leq P_{a v} .
\end{aligned}
$$

We solve the above Problem via the Lagrange duality method. First, we write the Lagrangian of above problem as

$$
\begin{aligned}
L(P, \lambda, \mu)= & \sum_{j=1}^{L} E\left[\log \left(1+g_{1} p_{j}\right)-\lambda p_{j}-\mu g_{0} p_{j} \mid \mathcal{R}_{j}\right] \\
& \times \operatorname{Pr}\left(\mathcal{R}_{j}\right)+\lambda P_{a v}+\mu Q_{a v}
\end{aligned}
$$

where $\lambda$ and $\mu$ are the nonnegative Lagrange multipliers associated with the ATP constraint and AIP constraint respectively. The Lagrange dual function $g(\lambda, \mu)$ is defined as

$\max _{p_{j} \geq 0 \forall j} \sum_{j=1}^{L} E\left[\log \left(1+g_{1} p_{j}\right)-\lambda p_{j}-\mu g_{0} p_{j} \mid \mathcal{R}_{j}\right] \operatorname{Pr}\left(\mathcal{R}_{j}\right)$

and the corresponding dual problem is

$$
\min _{\lambda \geq 0, \mu \geq 0} g(\lambda, \mu)+\lambda P_{a v}+\mu Q_{a v} .
$$

We first solve the optimization problem (6) with fixed $\lambda$ and $\mu$. To this end, we employ an algorithm similar to the Generalized Lloyd Algorithm (GLA) [11], [12] to design an optimal codebook for Problem (6), which is based on two optimality conditions: 1) optimum channel partitioning for a given codebook, also called the nearest neighbor condition
(NNC) in the context of traditional vector quantization (VQ), and 2) optimum codebook design for a given partition, also known as the centroid condition (CC) (in the context of VQ) [12]. GLA is usually initialized with a random choice of codebook, and then the above two conditions are iterated until some pre-specified convergence criterion is met. The same procedure is used here for designing an optimal quantizer $\mathcal{Q}$, but the design criterion for our case is minimizing the difference between the capacity with perfect CSI and the capacity with quantized power allocation under the given constraints. This amounts to designing an optimal power codebook $\mathcal{Q}$ that maximizes the Lagrangian function for quantized CSI, $\sum_{j=1}^{L} E\left[\log \left(1+g_{1} p_{j}\right)-\lambda p_{j}-\mu g_{0} p_{j} \mid \mathcal{R}_{j}\right] \operatorname{Pr}\left(\mathcal{R}_{j}\right)$. We call the corresponding quantized power allocation algorithm for a given $\lambda, \mu$ as a modified GLA.

In practice, this modifed GLA is implemented using a sufficiently large number of training samples (channel realizations for $\left.g_{0}, g_{1}\right)$. Beginning with a random initial codebook, one can design the optimal partitions using the fact that the optimal partitions satisfy

$$
\begin{aligned}
\mathcal{R}_{j}= & \left\{\left(g_{0}, g_{1}\right):\left(\log \left(1+g_{1} p_{j}\right)-\lambda p_{j}-\mu g_{0} p_{j}\right) \geq\right. \\
& \left.\left(\log \left(1+g_{1} p_{n}\right)-\lambda p_{n}-\mu g_{0} p_{n}\right), \forall n \neq j\right\}
\end{aligned}
$$

where $\mathcal{R}_{j}$ is the corresponding partition region for power level $p_{j}$ in the codebook, and ties are broken arbitrarily. Once the optimal partitions are designed, the new optimal power codebook is found by solving for $\operatorname{argmax}_{p_{j} \geq 0} E[\log (1+$ $\left.\left.g_{1} p_{j}\right)-\lambda p_{j}-\mu g_{0} p_{j} \mid \mathcal{R}_{j}\right] \operatorname{Pr}\left(\mathcal{R}_{j}\right), \forall j=1,2, \ldots, L$. Given a partition, this optimization problem is convex and by using the KKT conditions, one can obtain the optimal power as $\max \left(p_{j}^{*}, 0\right)$, where $p_{j}^{*}$ is the solution to the equation $E\left[\frac{g_{1}}{1+g_{1} p_{j}}-\left(\lambda+\mu g_{0}\right) \mid \mathcal{R}_{j}\right]=0$. These two steps are repeated until the resulting ergodic capacity converges within a prespecified accuracy. One needs to note that GLA cannot in general guarantee global optimality, since the two optimality conditions (NNC and CC) mentioned above are just necessary conditions [12]. Thus it is possible that the resulting quantizer is only locally optimal. While convergence of our modified GLA follows immediately by noting that the Lagrangian $\sum_{j=1}^{L} E\left[\log \left(1+g_{1} p_{j}\right)-\lambda p_{j}-\mu g_{0} p_{j} \mid \mathcal{R}_{j}\right] \operatorname{Pr}\left(\mathcal{R}_{j}\right)$ is non-decreasing at each iteration and is upper bounded (due to finite average transmit power and average interference constraints), it is important to state a more formal result along the lines of [13]. Since GLA is initialized with a random codebook and the optimal partitions and codevectors are found using training samples drawn from empirical distributions, it is crucial that GLA is globally convergent with respect to the choice of initial codebooks and empirically consistent. For more formal definitions of these two properties, see [13]. Under the assumption of absolutely continuous fading distributions for $g_{0}, g_{1}$ and mild regularity assumptions satisfied by these distributions, one can show that the modified GLA satisfies the conditions for global convergence and empirical consistency stated in [13] and thus we have the following result:

Theorem 2: The modified GLA that solves the optimization problem (6) satisfies the global convergence and empirical consistency properties of [13].

Next, we present some useful properties of the optimal power solutions obtained via the modified GLA. We use the 
partitions $\mathcal{R}_{1}, \ldots, \mathcal{R}_{L}$ and the corresponding power levels $p_{1}, \ldots, p_{L}$ to denote the convergent optimal solutions.

Lemma 1: Given partitions $\mathcal{R}_{1}, \ldots, \mathcal{R}_{L}$ and the corresponding power level $p_{1}, \ldots, p_{L}$, (where $\mathcal{R}_{j}$ and $\mathcal{R}_{j+1}, \forall j \in$ $\{1, \ldots, L-1\}$ are adjacent regions and $p_{j} \neq p_{j+1}$ ), the boundary between any two adjacent regions $\mathcal{R}_{j}$ and $\mathcal{R}_{j+1}$ is given by

$$
g_{1}=\frac{e^{\left(\lambda+\mu g_{0}\right)\left(p_{j}-p_{j+1}\right)}-1}{p_{j}-p_{j+1} e^{\left(\lambda+\mu g_{0}\right)\left(p_{j}-p_{j+1}\right)}} .
$$

When $\mu \neq 0, g_{1}$ is a monotonically increasing convex function of $g_{0}$ and as $g_{1} \rightarrow \infty$, we have $g_{0} \rightarrow$ $\frac{1}{\mu}\left(\frac{\log \left(\frac{p_{j}}{p_{j+1}}\right)}{\operatorname{Rem}^{p} \overline{\bar{a}} \mathrm{P}_{k+1}+1}-\lambda\right)$ In case $\lambda$. active and the ATP constraint is satisfied with equality. In this case, the boundary between any two adjacent regions $\mathcal{R}_{j}$ and $\mathcal{R}_{j+1}$ becomes $g_{1}=\frac{e^{\lambda\left(p_{j}-p_{j+1}\right)}-1}{p_{j}-p_{j+1} e^{\lambda\left(p_{j}-p_{j+1}\right)}}$. Clearly, Problem (4) reduces to an ergodic capacity maxmization problem with quantized channel information. For the narrowband case, it becomes a scalar quantization problem involving quantizing $g_{1}$ only. Note that while for the narrowband case, this no longer pertains to a cognitive radio problem, the properties of the optimal quantized power allocation scheme are still important for the wideband case $(M>1)$. This is due to the fact that in the wideband case, it is possible that for a specific (say the $i$-th) channel, the AIP constraint is inactive $\left(\mu_{i}>0\right)$ while $\lambda>0$. See Section IV-B for further details.

We now give an example to illustrate what the optimum partition regions actually looks like. For this example, $g_{0}$ and $g_{1}$ are both exponentially distributed (Rayleigh fading) with unit mean and $L=4$ ( 2 bits of feedback). The optimum partition regions are as shown in Fig. 1 for $\lambda>0, \mu>0$, and the Figure for $\lambda>0, \mu=0$ can be found in [8].

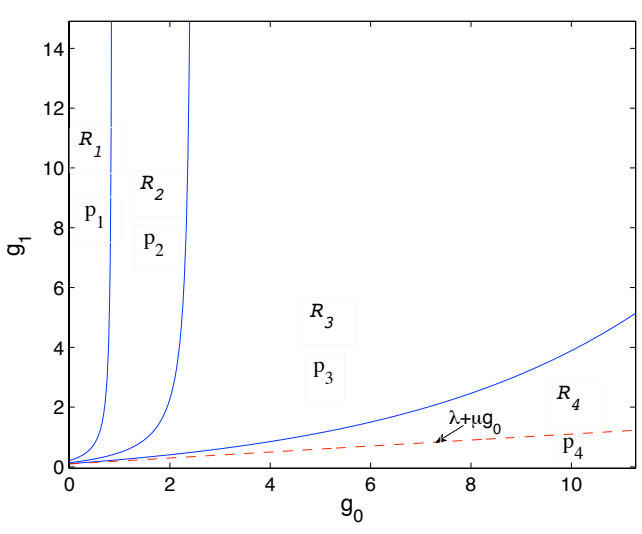

Fig. 1. The structure of optimum partition regions with $B=2$ bits feedback given $\lambda=\mu=0.1$.

Proposition 1: We obtain the following properties for the optimal quantized power levels where (as illustrated in Figure 1) the regions $\mathcal{R}_{1}, \mathcal{R}_{2}, \ldots$ etc. are sequentially numbered, with $\mathcal{R}_{1}$ being the region closest to the $g_{1}$ axis and $\mathcal{R}_{L}$ being the region closest to the $g_{0}$ axis. Note that these properties apply regardless of whether $\mu>0$ or $\mu=0$.

i) $p_{1}>\cdots>p_{L}$.

ii) All boundaries between any two adjacent partitions satisfy $g_{1}>\lambda+\mu g_{0}$ iii) Given B bits of feedback (or $L=2^{B}$ regions), for the first L-1 regions, we always have strictly positive power, i.e. $p_{1}>\cdots>p_{L-1}>0$, whereas $p_{L}$ is simply nonnegative, i.e. $p_{L} \geq 0$.

iv) When $\lambda+\mu \geq 1$ (note that if $\lambda=0, \mu \geq 1$ implies $Q_{\text {avg }}<1$, and if $\mu=0, \lambda \geq 1$ corresponds to $P_{\text {avg }}<$ 1 ), we always have $p_{L}=0$. In addition, when $L$ (the number of quantized regions) is sufficiently large, no matter what $\lambda, \mu$ is, $p_{L}$ must be 0 . Additionally, as $L \rightarrow$ $\infty$ the boundary between $\mathcal{R}_{L-1}$ and $\mathcal{R}_{L}$ approaches $g_{1}=\lambda+\mu g_{0}$ and $\lim _{L \rightarrow \infty} p_{L-1}=0$.

Remark 2: The above properties of optimal quantized power values are interesting for two reasons. From Property ii), it is clear that $\left(g_{0}, g_{1}\right) \in \mathcal{R}_{j}$ for $j=1,2, \ldots, L-1$ satisfy the property $g_{1}>\lambda+\mu g_{0}$ whereas for the region $\mathcal{R}_{L}$, this property may or may not be satisfied. Since the quantized power values in the first $L-1$ regions are strictly positive, it is easy to relate this property to the corresponding property of the full CSI based optimal power value which is strictly positive if and only if $g_{1}>\lambda^{f}+\mu^{f} g_{0}$. Also, as $L \rightarrow \infty$, the boundary between $\mathcal{R}_{L-1}$ and $\mathcal{R}_{L}$ approaches $g_{1}=\lambda+\mu g_{0}$, thus making this relationship between the quantized power allocation scheme and the full CSI power allocation scheme stronger.

Finally, Property iv) allows one to obtain an approximate quantized power allocation scheme (AQPA) for large $L$ by setting $p_{L}=0$ and taking the limit as $p_{L-1} \rightarrow 0$. This is particularly useful as the modified GLA becomes computationally intensive for large $L$, whereas AQPA provides a performance that is extremely close to that of the modified GLA, while requiring very little computation. A detailed description of the AQPA is provided in Sec. IV-C followed by illustrative numerical simulations in Sec. V.

Based on the above results, we can solve for the optimal quantized power values given a partition $\mathcal{R}_{1}, \mathcal{R}_{2}, \ldots, \mathcal{R}_{L}$. This is equivalent to solving the following set of nonlinear equations for $p_{1}, p_{2}, \cdots, p_{L}$ :

$$
\left\{\begin{array}{l}
E\left[\frac{g_{1}}{1+g_{1} p_{j}}-\left(\lambda+\mu g_{0}\right) \mid \mathcal{R}_{j}\right]=0, \quad j=1, \ldots, L \\
p_{L}=\max \left(0, p_{L}\right)
\end{array}\right.
$$

where if $\mu \neq 0, \quad E\left[\frac{g_{1}}{1+g_{1} p_{j}}-\left(\lambda+\mu g_{0}\right) \mid \mathcal{R}_{j}\right]=$ $\int_{c_{j}}^{\infty} \int_{r_{j-1}}^{r_{j}}\left(\frac{g_{1}}{1+g_{1} p_{j}}-\left(\lambda+\mu g_{0}\right)\right) f\left(g_{0}\right) f\left(g_{1}\right) d g_{0} d g_{1}$, with $c_{j}=$ $\frac{e^{\lambda\left(p_{j}-p_{j}+1\right)}-1}{p_{j}-p_{j+1} e^{\lambda\left(p_{j}-p_{j+1}\right)}}, j=1, \ldots, L-1, c_{L}=0$ and $r_{j}=\frac{1}{\mu}\left(\frac{\log \frac{p_{j} * g_{1}+1}{p_{j+1} * g_{1}+1}}{p_{j}-p_{j+1}}-\lambda\right), j=1, \ldots, L-1, r_{0}=$ $0, r_{L}=\infty$. When $\mu=0, E\left[\frac{g_{1}}{1+g_{1} p_{j}}-\left(\lambda+\mu g_{0}\right) \mid \mathcal{R}_{j}\right]=$ $\int_{c_{j}}^{c_{j-1}}\left(\frac{g_{1}}{1+g_{1} p_{j}}-\lambda\right) f\left(g_{1}\right) d g_{1}$, with $c_{0}=\infty$. Equation (10) can be solved efficiently by a nonlinear equation solver.

Now that we have an algorithm based on the modified GLA for solving the (possibly locally optimal) quantized power values for fixed $\lambda$, $\mu$, we can go back to solving the dual problem (7) for finding optimal values $\lambda$ and $\mu$. To this end, we exploit a similar method to the full CSI case in Sec. III to obtain optimal values $\lambda$ and $\mu$ numerically by solving the associated KKT conditions. One can thus repeat the above two steps by solving (6) and (7) iteratively until a satisfactory convergence criterion is met. A formal 
algorithmic format for this procedure is provided for the more general wideband $(M>1)$ case next.

\section{B. Wideband spectrum-sharing case}

The above algorithm for the narrowband case can be easily extended to the wideband case corresponding to the original Problem (2). For this scenario, the Lagrangian function is

$$
\begin{aligned}
L(P, \lambda, \mathbf{u}) & =\sum_{j=1}^{L}\left(\frac{1}{M} \sum_{i=1}^{M} E\left[\log \left(1+g_{1}^{i} p_{i j}\right) \mid \mathcal{R}_{j}\right]\right) \operatorname{Pr}\left(\mathcal{R}_{j}\right) \\
& -\lambda\left(\sum_{j=1}^{L}\left(\frac{1}{M} \sum_{i=1}^{M} E\left[p_{i j} \mid \mathcal{R}_{j}\right]\right) \operatorname{Pr}\left(\mathcal{R}_{j}\right)-P_{a v}\right) \\
& -\sum_{i=1}^{M} \mu_{i}\left(\sum_{j=1}^{L} E\left[g_{0}^{i} p_{i j} \mid \mathcal{R}_{j}\right] \operatorname{Pr}\left(\mathcal{R}_{j}\right)-Q_{a v, i}\right)
\end{aligned}
$$

where $\lambda$ and $\mu_{i}$ are the nonnegative Lagrange multipliers associated with the ATP constraint and $i$-th AIP constraint respectively. The Lagrange dual function $g\left(\lambda,\left\{\mu_{i}^{\prime}\right\}\right)$ is defined as

$$
\begin{aligned}
\max _{p_{i j} \geq 0} & \frac{1}{M} \sum_{i=1}^{M} \sum_{j=1}^{L} E\left[\log \left(1+g_{1}^{i} p_{i j}\right)-\lambda p_{i j}\right. \\
& \left.-\mu_{i}^{\prime} g_{0}^{i} p_{i j} \mid \mathcal{R}_{j}\right] \operatorname{Pr}\left(\mathcal{R}_{j}\right)
\end{aligned}
$$

where $\mu_{i}^{\prime}=M \mu_{i}, \forall i$, and the dual problem is

$$
\min _{\lambda \geq 0, \mu_{i}^{\prime} \geq 0, \forall i} g\left(\lambda,\left\{\mu_{i}^{\prime}\right\}\right)+\lambda P_{a v}+\sum_{i=1}^{M} \frac{\mu_{i}^{\prime}}{M} Q_{a v, i} .
$$

Similar to the narrowband case, we first consider the Problem (12) to obtain $g\left(\lambda,\left\{\mu_{i}^{\prime}\right\}\right)$ with given $\lambda$ and $\left\{\mu_{i}^{\prime}\right\}$. Denote by $\mathcal{R}_{j}^{i}$ the $j$-th quantization region for the $i$-th band where $\bigcup_{i=1}^{M} \mathcal{R}_{j}^{i}=\mathcal{R}_{j}$. Then problem (12) can be decomposed into $\mathbf{M}$ parallel subproblems, where for each band $i, i=1, \ldots, M$,

$$
\max _{p_{i j} \geq 0 \forall j} \sum_{j=1}^{L} E\left[\log \left(1+g_{1}^{i} p_{i j}\right)-\lambda p_{i j}-\mu_{i}^{\prime} g_{0}^{i} p_{i j} \mid \mathcal{R}_{j}^{i}\right] \operatorname{Pr}\left(\mathcal{R}_{j}^{i}\right)
$$

is defined as the sub-dual function $g_{i}\left(\lambda, \mu_{i}^{\prime}\right)$ and $g\left(\lambda,\left\{\mu_{i}^{\prime}\right\}\right)=$ $\frac{1}{M} \sum_{i=1}^{M} g_{i}\left(\lambda, \mu_{i}^{\prime}\right)$. This kind of duality method is also known as the 'dual decomposition algorithm' [14]. Since each subproblem above is similar to Problem (6) for the narrowband case, they can be similarly solved by using a modified GLA. $\lambda$ and $\left\{\mu_{i}^{\prime}\right\}$ can also be obtained in a manner similar to the narrowband case. These two steps are then repeated until a satisfactory convergence criterion is met. Due to the increased complexity resulting from the presence of multiple bands, we provide below a formal description of the overall optimization algorithm (Algorithm 1) for solving (4).

\section{Algorithm 1:}

1) Let $\lambda=0$, then all $\mu_{i}^{\prime}, i=1, \ldots, M$ must satisfy $\mu_{i}^{\prime}>0$. Starting with some random initial power codebook, for each $i$, find $\mu_{i}^{\prime}$ by solving $\sum_{j=1}^{L} E\left[g_{0}^{i} p_{i j} \mid \mathcal{R}_{j}\right] \operatorname{Pr}\left(\mathcal{R}_{j}\right)=$ $Q_{a v g}^{i}$ and then obtain the corresponding (locally) optimal power codebook $\left\{p_{i 1}, \ldots, p_{i L}\right\}$ using a modified GLA. Repeat these two steps until convergence resulting in a power codebook $\left\{\mathbf{P}_{1}, \ldots, \mathbf{P}_{L}\right\}$. With this codebook, if $\sum_{j=1}^{L}\left(\frac{1}{M} \sum_{i=1}^{M} E\left[p_{i j} \mid \mathcal{R}_{j}\right]\right) \operatorname{Pr}\left(\mathcal{R}_{j}\right) \leq P_{a v g}$, it's an optimal codebook and stop; otherwise go to step 2 ).
2) If 1) is not satisfied, we must have $\lambda>0$. For a given $\lambda$, for each $i$, use the modified GLA to find an optimal power codebook first with $\mu_{i}^{\prime}=0$. If $\sum_{j=1}^{L} E\left[g_{0}^{i} p_{i j} \mid \mathcal{R}_{j}\right] \operatorname{Pr}\left(\mathcal{R}_{j}\right) \leq Q_{a v g}^{i}$, then the corresponding optimal codebook $\left\{p_{i 1}, \ldots, p_{i L}\right\}$ (obtained via the modified GLA) is an optimal solution for this $i$-th subproblem, otherwise, $\mu_{i}^{\prime}>0$, and can be found by solving $\sum_{j=1}^{L} E\left[g_{0}^{i} p_{i j} \mid \mathcal{R}_{j}\right] \operatorname{Pr}\left(\mathcal{R}_{j}\right)=Q_{\text {avg }}^{i}$. Find the corresponding optimal codebook entry $\left\{p_{i 1}, \ldots, p_{i L}\right\}$ for the $i$-th subband, and then use this codebook to find an updated value of $\lambda$ by solving $\sum_{j=1}^{L}\left(\frac{1}{M} \sum_{i=1}^{M} E\left[p_{i j} \mid \mathcal{R}_{j}\right]\right) \operatorname{Pr}\left(\mathcal{R}_{j}\right)=P_{a v g}$. Repeat these steps until convergence and the final codebook will be an optimal codebook for the wideband spectrum sharing problem (4).

\section{Approximate Quantized Power Allocation Algorithm (AQPA)}

Although an offline algorithm, the complexity of modified GLA for determining the optimal quantized power is very high for even a moderately large value of $L$. This is due to the fact that the optimal channel partitions and the corresponding optimal power codebook are obtained via empirically generating a large number of channel realizations as training samples. As $L$ increases, the number of training samples required will also increase. Thus, we use part iv) of Lemma 1 to derive a low-complexity suboptimal scheme for implementing the modified GLA for large $L$ values. Below we describe this scheme for the narrowband case. A similar scheme for the wideband case can be designed accordingly.

Note that part iv) of Lemma 1 states that as $L \rightarrow \infty$, $p_{L}=0$ and $p_{L-1} \rightarrow 0$. Applying these approximations to (10) allows us to obtain an approximate but computationally efficient algorithm (called approximate quantized power allocation algorithm (AQPA)) for large $L$. AQPA first solves $E\left[\frac{g_{1}}{1+g_{1} p_{L-1}}-\left(\lambda+\mu g_{0}\right) \mid \mathcal{R}_{L-1}\right]=0$ for $p_{L-2}$ by substituting $p_{L}=0$ and taking the limit $p_{L-1} \rightarrow 0$, which, if $\mu>0$, is equivalent to solving $\int_{\lambda}^{\infty} \int_{\frac{1}{\mu}\left(\frac{g_{1}-\lambda}{\mu}\right.}^{\left.\frac{\log \left(1+g_{1} p_{L-2}\right)}{p_{L}-2}-\lambda\right)}\left(g_{1}-\right.$ $\left.\left(\lambda+\mu g_{0}\right)\right) f\left(g_{0}\right) f\left(g_{1}\right) d g_{0} d g_{1}=0$ for $p_{L-2}$. When $\mu=0$, it is equivalent to solving for $p_{L-2}$ from $\int_{\lambda}^{\frac{e^{\lambda p_{L-2}-1}}{p_{L-2}}}\left(g_{1}-\right.$ d) $f\left(g_{1}\right) d g_{1}=0$. Note that the above equations (for both $\mu>0$ and $\mu=0$ ) involve only one variable: $p_{L-2}$ and are thus straightforward to solve. One can then recursively compute $p_{L-3}, p_{L-4}, \ldots$, by using the optimality conditions for the regions $\mathcal{R}_{L-2}, \mathcal{R}_{L-3}, \ldots$, respectively, in the reverse direction. These equations can be solved by appropriate nonlinear equation solvers and do not require the use of large number of training samples. Thus AQPA has a substantially reduced computational complexity and is applicable to large number of feedback bits. Note however, as this is an approximate algorithm only, the performance of this algorithm becomes comparable to modified GLA only for large values of $L$. Numerical results presented in the next section illustrate that AQPA performs extremely well for $L \geq 16$.

\section{NUMERICAL RESULTS}

In this section, we will evaluate the performance of the designed power allocation strategies via numerical simulations. We use a wideband spectrum sharing system with one $\mathrm{SU}$ and $M$ independent frequency bands (each band is 
originally licensed to a PU), where all the channels involved are Rayleigh fading. For each simulation, 100,000 randomly generated channel realizations for each $\mathbf{g}_{0}$ or $\mathbf{g}_{1}$ are used.

Fig. 2 shows the capacity performance of SU sharing the spectrum with four PUs $(M=4)$ (wideband spectrum sharing case) under AIP constraint thresholds $\left(Q_{a v, 1}, Q_{a v, 2}, Q_{a v, 3}, Q_{a v, 4}\right)=(-10 \mathrm{~dB},-5 \mathrm{~dB}, 0 \mathrm{~dB}, 5 \mathrm{~dB})$, and illustrates the effect of increasing the number of feedback bits on the capacity performance. For comparison, we also plot the corresponding capacity performance with full CSI. The striking observation from Fig. 2 is that introducing one extra bit of feedback substantially reduces the gap with capacity based on perfect CSI. This property is not very obvious when $P_{a v}$ is small, for example when $P_{a v} \leq 0 \mathrm{~dB}$. But with increasing $P_{a v}$, it becomes more pronounced. To be specific, at $P_{a v}=10 \mathrm{~dB}$, with 1 bit, 2 bits and 3 bits of feedback, the percentage capacity loss is approximately $17.38 \%, 5.49 \%$ and $1.46 \%$ respectively, and only 3 bits feedback can result in secondary ergodic capacity very close to that with full CSI. This is very encouraging since only a small number of bits of feedback are required to achieve close performance to the full CSI case.

In Fig. 3 we compare the performance of AQPA with modified GLA, where SU shares the spectrum with two PUs $(M=2)$ and AIP constraint thresholds $\left(Q_{a v, 1}, Q_{a v, 2}\right)=(-5$ $\mathrm{dB}, 0 \mathrm{~dB})$. It illustrated that with the same number of bits of feedback, the gap between AQPA and modified GLA becomes smaller as $L$ increases. For example, when $P_{a v}=$ $10 \mathrm{~dB}$, the capacity loss by using AQPA instead of GLA is about $7.60 \%, 3.30 \%$ and $0.90 \%$ for 2 bits, 3 bits and 4 bits feedback respectively. It is clearly seen that AQPA with 4 bits feedback can almost approach the 4 bits GLA performance, but with much faster speed.

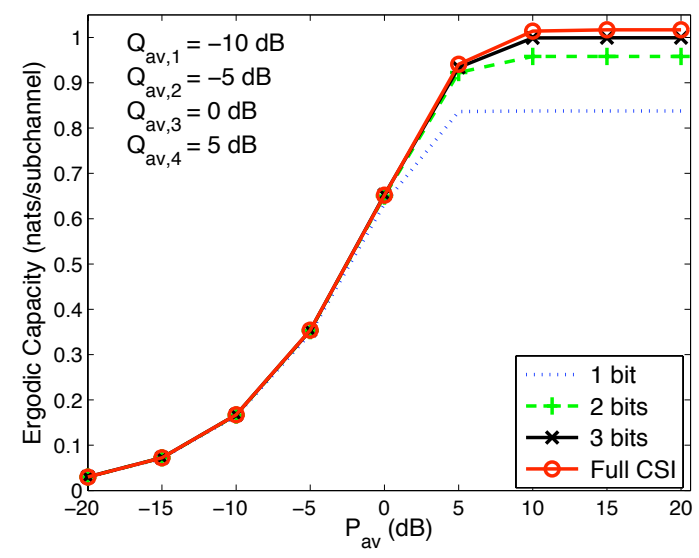

Fig. 2. SU Ergodic capacity with quantized power allocation (GLA) with four PUs $(M=4)$

\section{CONCLUSIONS AND EXTENSIONS}

We have derived quantized power allocation algorithms for a wideband spectrum sharing system with one secondary user and multiple primary users, each licensed to use a separate band, each band modelled as a block fading channel. The objective has been to maximize the SU ergodic capacity under an average sum transmit power constraint and individual average interference constraints at the PU receivers.

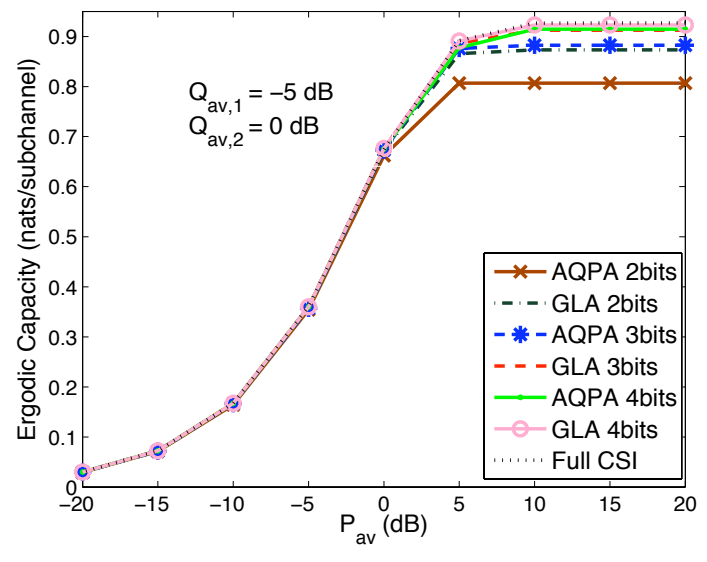

Fig. 3. Capacity performance of AQPA with two PUs $(M=2)$.

Generalized Lloyd type algorithms have been derived and various properties of the quantized power allocation laws have been presented. Future work will include deriving more comprehensive properties of the quantized power allocation laws, and deriving expressions for asymptotic capacity loss with quantized power allocation, and consideration of multiple PU's in each band.

\section{REFERENCES}

[1] A. Ghasemi and E. S. Sousa, "Fundamental limits of spectrum-sharing in fading environments," IEEE Trans. Wireless Commun., vol. 6, no. 2, pp. 649-658, Feb. 2007.

[2] J. Mitola, III, "Cognitive radio for flexible mobile multimedia communications," IEEE Int. Workshop on Mobile Multimedia Commun. $($ MoMuC), pp. 3-10, Nov. 1999.

[3] A. Goldsmith, S. A. Jafar, I. Maric and S. Srinivasa, "Breaking spectrum gridlock with cognitive radios: an information theoretic perspective," Proc. IEEE, vol. 97, no. 5, pp. 894-914, May 2009.

[4] J. M. Peha, "Sharing spectrum through spectrum policy reform and cognitive radio," Proc. IEEE, vol. 97, no. 4, pp. 708-719, Apr. 2009.

[5] R. Zhang, "On peak versus average interference power constraints for protecting primary users in cognitive radio networks," IEEE Trans. Wireless Commun., vol. 8, no. 4, pp. 2112-2120, Apr. 2009.

[6] X. Kang, Y. Liang, A. Nallanathan, H. K. Garg and R. Zhang, "Optimal power allocation for fading channels in cognitive radio networks: Ergodic capacity and outage capacity," IEEE Trans. Wireless Commun., vol. 8, no. 2, pp. 940-950, Feb. 2009.

[7] M. Gastpar, "On capacity under received-signal constraints," Annual Allerton Conf. on Commun., Cont. and Comput., 2004.

[8] —, "Spectrum sharing in cognitive radio with quantized channel information," [Online]. http://arxiv.org/abs/1006.0619.

[9] L. Musavian and S. Aissa, "Capacity and power allocation for spectrumsharing communications in fading channels," IEEE Trans. Wireless Commun., vol. 8, no. 1, pp. 148-156, Jan. 2009.

[10] L. Musavian and S. Aissa, "Fundamental capacity limits of cognitive radio in fading environments with imperfect channel information," IEEE Trans. Commun., vol. 57, no. 11, pp. 3472-3480, Nov. 2009.

[11] Y. Linde, A. Buzo and R. Gray, "An algorithm for vector quantizer design," IEEE Trans. Commun., vol. 28, no. 1, pp. 84-95, Jan. 1980

[12] A. Gersho and R. Gray, "Vector quantization and signal compression," Kluwer Publishers, 1992.

[13] M. Sabin and R. Gray, "Global convergence and empirical consistency of the generalized Lloyd algorithm," IEEE Trans. Inf. Theory, vol. 32, no. 2, pp. 148-155, Mar. 1986.

[14] L. Zhang, Y. Xin and Y. Liang, “Optimal power allocation for multiple access channels in cognitive radio networks," Proc. IEEE Veh. Tech. Conf., pp. 1550-2252, May 2008.

[15] R. Zhang, S. Cui and Y. Liang "On ergodic sum capacity of fading cognitive multiple-access and broadcast channels," IEEE Trans. Inf. Theory, vol. 55, no. 11, pp. 5161-5178, Nov. 2009.

[16] K. Huang and R. Zhang "Cooperative feedback for multiantenna cognitive radio networks ," [Online]. http://arxiv.org/abs/0911.2952.

[17] R. Zhang, Y-C. Liang and S. Cui, "Dynamic resource allocation in cognitive radio networks," IEEE Sig. Proc. Magaz., vol. 27, no. 3, pp. 102-114, Mar. 2010. 